Artículo recibido:

30 de junio del 2012.

Evaluado:

7 de agosto del 2012.

Aceptado:

28 de agosto del 2012.

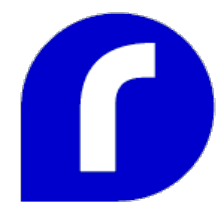

Luis Alexánder González

\section{La seguridad alimentaria frente a la internacionalización del comercio}

\section{RESUMEN}

Los grandes actores agrícolas (Estados Unidos, la Unión Europea y los grandes países periféricos) evolucionan hacia una liberalización progresiva del comercio mundial. La ponderación que las políticas agrícolas establecen entre apertura y protección constituye el marco ideal para el estudio de la problemática emergencia de la libre circulación de productos agrícolas, pues ella puede caracterizar algunos impasses socio-políticos relacionados con la seguridad alimentaria. La tendencia hacia la liberalización puede explicar por qué para la comunidad internacional los acuerdos comerciales son uno de los medios empleados por algunos países para obtener la apertura de los mercados.

Palabras Clave

Políticas agrícolas, agricultura, liberalización, alimentos

\section{Food Security and the Internationalization of Trade}

ABSTRACT

The big agricultural actors at a worldwide level (United States of America, the European Union, and a big group of peripheral countries) are evolving in a progressive liberalization of the world trade. The agricultural policies' balance of liberalization and protection elements constitutes the ideal framework to analyze the problematic emergence of the free circulation of food. It can additionally characterize some socio-political impasses related to the food security. This tendency towards liberalization can explain why for the international community trade agreements are used by some countries so to get market openings.

\section{KEY WORDS} Agricultural policies, agriculture,
liberalization, food
Doctor en Derecho de la Universidad francesa de Panthéon-Sorbonne, París I. Máster en Derecho europeo de la agricultura y de las industrias alimentarias de la misma universidad. El autor obtuvo su título de abogado en la Universidad Nacional de Colombia.

Correo electrónico: luis.gonzalez-martin@malix.univ-paris1.fr 


\section{La seguridad alimentaria frente a la internacionalización del comercio*}

\section{Introducción}

Profesionales de diversas disciplinas concentran su atención en los efectos de los procesos de liberalización del comercio internacional de productos agroalimenticios sobre el bienestar humano, la seguridad alimentaria y las políticas agrícolas nacionales. En el Sur, los países periféricos muestran tendencias que rápidamente se asimilan a las crisis de salud pública a las cuales se confrontan las sobrealimentadas poblaciones de los países industrializados. La noción tradicional de centro y periferia se desdibuja. En los países ricos crecen los archipiélagos periféricos empobrecidos, como en los países pobres surgen "sucursales" de la opulencia de los centros (Vargas, 2006).

En adelante se empleará indistintamente los términos países periféricos y en vía de desarrollo, sin olvidar el carácter colonial de la historia y desarrollo de este último concepto, como bien lo describe Rist (2007) en su obra. Por otra parte, la explosión en los índices de enfermedades tales como diabetes, cáncer o enfermedades cardíacas, asociadas a la obesidad y a dietas ricas en calorías pero pobres desde el punto de vista nutricional, se explica desde una paradoja producida por la liberalización del mercado de productos agroalimenticios: una disponibilidad creciente a una dieta rica en materias grasas y en calorías a precios y calidad bajos, producto de una agricultura intensiva practicada a nivel mundial, dieta a la cual acceden, en las llamadas grandes
* Un agradecimiento a Kathryn Peake por su colaboración 
superficies de distribución, los sectores sociales más pobres, resultado: malnutrición y obesidad tanto en el norte como en el sur.

Irónicamente, similares deficiencias de micro nutrientes están asociadas tanto con el hambre como con las dietas que producen la obesidad, la anemia y la deficiencia de vitamina A, causando ceguera en los niños menores de cinco años [...] Hoy en día, una dieta rica en grasa se encuentra disponible para los sectores sociales de más bajos niveles de ingresos, propiciando la difusión de la malnutrición y la obesidad en las clases económicamente más pobres de la población. En Brasil y México, al igual que en los EE.UU. y Australia, la obesidad es una enfermedad de los pobres más que de los ricos (IATP, 2007, p. 8).

Ahora bien, si la obesidad es un problema global mayor, el hambre se instala en un dramático proceso simultáneo. Más de 1000 millones de personas subalimentadas a nivel global; 28 millones en Europa del Este y en Rusia, 9 millones en los países industrializados de Europa Occidental y en los Estados Unidos (Charvet, 2010). El aumento del desempleo y la precariedad en las condiciones de trabajo también generan un impacto sobre el número de pobres y de malnutridos (UNICEF, 2006). En Estados Unidos los gobiernos, demócratas o republicanos reconducen de manera sucesiva el programa de distribución de bonos alimentarios Food stamps que favorece a más de cuarenta millones de personas (Oliveira, 2005; Thoyer, 1995). Mientras en Francia los Restaurants du cœur acogen a miles de personas cada invierno en campañas que se integran a dispositivos institucionales aún más amplios (AFDR, 2011).

Con el fin de intentar dar una descripción plausible de los elementos en el sector de la producción agrícola, ligados a este doble fenómeno de consumo deficitario de alimentos, un análisis adecuado de los sistemas agroalimentarios locales-nacionales supone el estudio del funcionamiento del sistema agroalimentario mundial, y de cómo modifica sus relaciones con los mercados y estructuras económicas nacionales (Bonnamour, 2006). Así, se desea que un esfuerzo de síntesis contemple las dos primeras etapas que constituyen este proceso global: producción e intercambio, que forman junto a la distribución y el consumo de productos agrícolas y agroalimentarios un verdadero silogismo de causa efecto, un silogismo constitutivo a escala mundial (Marx, 1953). 
El individuo que antes contribuía a la producción agrícola, se ha visto abocado, por necesidad, a abandonar su labor de productor para ir a engrosar la población asalariada (y no asalariada) de las ciudades, y a participar en una nueva función de distribución y de consumo de productos que provienen de un proceso de producción y transformación cada vez más mundializado'.

Las estructuras de distribución, que antes eran enteramente determinadas por la producción nacional o local, sostienen hoy en día una relación antagónica en donde la antigua correlación se ve enteramente invertida. Actualmente, es posible afirmar que la tendencia hacia la concentración en el campo de la distribución en manos de empresas multinacionales es el factor fundamental que determina la estructura de la producción. Ahora bien, ¿podrá esta estructura alimentar la población mundial? Población compuesta en un futuro próximo por nueve mil millones de seres humanos cada vez más urbanos y de los cuales más de 1000 millones padecen de hambre (Charvet, 2010).

En relación con el anterior contexto, se delinea en la investigación las tendencias de los sistemas alimentarios en el sector de la producción, se definen el marco económico-político, las modalidades de regulación estatal - la política macro-económica-, la política agrícola, la política de comercio exterior, con el fin de entender los móviles que preceden las negociaciones y los acuerdos comerciales de orden librecambista que se sostienen a nivel global. Para tal fin, se abordará la naturaleza de la intervención estatal en la producción agrícola, particularmente en los EE.UU. y en Europa, para luego, de forma general, abordar la heterogénea y deficitaria intervención estatal para el caso de América Latina, identificando tendencias, similitudes y contrastes con algunas de las regiones del mundo.

\section{Políticas agrícolas y comercio internacional: grandes tendencias en EE.UU., en la Unión Europea y en Latinoamérica}

Se presenta en este apartado un conjunto de hechos que permite comprender el cómo formular la cuestión agroalimentaria a nivel nacional e internacional. Para esto, se esbozará cómo los estados y las estructuras político económicas regionales han establecido los medios, técnicas y formas de organización adecuadas para la producción de alimentos en un contexto global. Ahora bien, si la función esencial de un sistema social, político, jurídico o económico es asegurar la reproducción y el bienestar de los individuos que lo componen, es claro que la seguridad alimentaria será uno de los primeros objetivos por alcanzar, que además sirve de referente de corrección para el presente análisis.
1. Gracias a la inversión masiva internacional, los productos agroalimentarios circulan determinando flujos cada vez más rápidos en el tiempo y progresivamente integrados en el espacio. La circulación se convierte ante todo en la expansión de la potencia de un modo de producción intensivo dirigido hacia lo internacional. 


\section{La estrategia agrícola estadounidense}

Los bloques del este y del oeste, 20 años después de la caída del Muro de Berlín, han sido sustituidos por una situación económica mundial dominada por los Estados Unidos, la Unión Europea, Japón, y por la consolidación progresiva de las llamadas economías emergentes: China, Rusia, India y Brasil. Este proceso de fragmentación del espacio-mundo se acompaña por uno de regionalización, del cual hace parte el NAFTA, North American Free Trade Agreement, establecido en 1992-1993; el acuerdo engloba una zona de libre comercio que comprende los Estados Unidos de América, Canadá y México, para un total de 440 millones de habitantes, representando una cuarta parte del PIB mundial y sede de un número impresionante de multinacionales cuya participación equivale a otra cuarta parte. Se trata de una reestructuración favorable a los intereses de los Estados Unidos, particularmente a aquellos del sector agrícola. Por ejemplo, las exportaciones hacia México, especialmente en maíz, aumentan en 323\% comparando el promedio anual de 1,6 millones de toneladas en el período anterior a la implementación del acuerdo (19901993), con el periodo posterior a dicho acuerdo cuando, desde 1996, se exportaron 5 millones de toneladas anuales de este cereal (Nadal y Wise, 2004).

Los Estados Unidos cuentan con 400 millones de hectáreas de superficie agrícola utilizada, dos millones de estructuras productivas (1,8 millones de familias en la American Farm Bureau), cada una de cerca de 200 hectáreas en promedio (Revel \& Ribaud, 1981; Thoyer, 1998), las cuales contribuyen en $22 \%$ del valor de las exportaciones agrícolas mundiales. Este sector también emplea 3\% del total de la población activa de ese país; "de 13,5 millones en 1910 la población agrícola disminuye a alrededor de tres millones en el año 2000" (McDonald, 2006, pp. 6-9). A pesar del poco peso en términos demográficos, la contribución del complejo agroindustrial al PIB es de aproximadamente $14 \%$, lo que hace de este sector un actor económico importante (Thoyer, 1998).

La política estadounidense se ha centrado, incluso antes de la independencia, en cuestiones relacionadas con lo agroalimentario: la negativa de pagar tasas al tesoro inglés sobre el té propiciaría la creación de la Boston Tea Party en 1773 y el comienzo de la guerra de independencia. Posteriormente, la Guerra Civil se desarrolla como consecuencia de la disputa norte-sur en relación con la mecanización de la producción del algodón (Marx \& Engels, 2002). En 1862, el presidente Abraham Lincoln firma la Morill Act, que dará a cada uno de los estados federados un terreno para construir un campus universitario dotado de un centro de investigación agrícola. Así aparecerían los cincuenta Land Grant College en donde ha sido concebida la política agrícola estadounidense plasmada en las sucesivas leyes agrícolas o Farm Bills. 
A comienzos del siglo $X X$ el mercado decreta la inviabilidad para la pequeña agricultura, lo que genera un desplazamiento masivo de la población rural a zonas urbanas y periurbanas. La propiedad en forma de grandes latifundios y en manos de la agroindustria se consolidaría. El aumento de la productividad basada en la gestión optimizada del agua, la mecanización y el uso de fertilizantes produce que la granja familiar y la pequeña producción independiente desaparezcan rápidamente.

El ejemplo clásico es el de California. La construcción de grandes represas y sistemas de riego fue un factor determinante en la transición de la pequeña explotación familiar a la producción mercantil a gran escala. La concentración de la propiedad fue acompañada por mejoras en la tecnología y un aumento de la productividad. (Pisani, 1984 , p. xi)

\section{Exportaciones: factor clave de la agricultura estadounidense}

En 1933, en la época del New Deal, se establece por primera vez una política intervensionista con la firma de la Agricultural Adjustement Act por el presidente Franklin Delano Roosevelt, la que a su vez, posteriormente inspirase las políticas europeas proteccionistas de posguerra (Thoyer, 1998; IATP, 2007b).

Desde los años ochenta los EE.UU. son el mayor exportador agrícola del mundo. En 2008, las transacciones alcanzan los \$ 101 mil millones y el superávit comercial de productos agrícolas llega a $\$ 24,5$ mil millones; sin verse mayormente afectados por la crisis generada por la aparición a nivel mundial de productos en grandes cantidades provenientes de la Unión Europea, de Brasil, de Argentina y de Australia (Adam, 2006).

La política agrícola estadounidense ha estado, sobre todo a partir de 1972, basada sobre cuatro productos, maíz, soja, trigo (de los cuales son ellos los principales exportadores mundiales) y carne, sector en el cual se han convertido en líderes mundiales desde hace dos décadas (Thoyer, 1998; Charvet, 2010).

La política agrícola estadounidense alienta a los productores de maíz y soja para que provean al mejor precio a los productores de carne nacionales e internacionales, quienes a su vez satisfacen a los consumidores cada vez más numerosos en el sur emergente ${ }^{2}$. A largo plazo, la producción de carne será el sector privilegiado, pues es allí en donde la demanda global será más importante (IATP, 2007). Sin embargo, se prevé que para el año 2020 este sector será deficitario lo que conllevaría a un aumento sostenido de los precios.

2. El aumento de la demanda mundial de soja obedece a que muchos países han adoptado una parte del modelo de crianza estadounidense, crianza intensiva de aves de corral y de ganado, pero con una industria de alimentos concentrados inexistente 0 deficitaria. Así, se consolidan regiones en el mundo en donde se importa proteína vegetal para la alimentación de los animales. 
Este marco productivo supone la liberación de todo tipo de restricciones institucionales estatales a nivel internacional, así como la reducción de todo tipo de barreras al comercio a través de negociaciones que toman cuerpo en acuerdos comerciales internacionales, tratados de libre comercio, en particular aquellos firmados en el seno de la Organización Mundial del Comercio (OMC). La liberalización del comercio de productos agrícolas propiciaría, en los términos ideales planteados por el liberalismo económico, que el precio mundial fuese aquel establecido por el mayor y más sólido productor, es decir quien ya posee una ventaja comparativa consolidada históricamente.

La estrategia estadounidense frente al mercado internacional es puesta en marcha tanto por administraciones demócratas como republicanas, facilitada por un lado mediante el financiamiento de los productos agrícolas (Kaufman, 2010) y por otro, mediante la adopción de una legislación comercial que dota a sus exportadores de los elementos presupuestales para competir a nivel mundial. Como un ejemplo, los subsidios directos establecidos en 1985 a través del Export Enhancement Program, diseñado para combatir las prácticas de comercio de otros países consideradas como desleales. Los exportadores estadounidenses reciben de esta manera primas correspondientes a la diferencia entre el precio de compra en el mercado interno y el precio mundial al que se venden los diferentes productos (Blandford, 2008).

El Programa de Promoción de Mercados o Market Access Program (MAP) 1996, autoriza al United States Department of Agriculture (USDA) a contribuir financieramente a la promoción de productos en el extranjero; reservada para pequeñas y medianas empresas y para organizaciones profesionales. La ayuda cubre los gastos de prospección de mercado y de publicidad, centrándose en productos de alto valor agregado. Este programa se financia con recursos de la Commodity Credit Corporation (CCC) cuyo límite en términos de gastos anuales está fijado en 90 millones de dólares.

En relación con las garantías de crédito para la exportación correspondientes al corto y al mediano plazo (6 meses a 10 años GSM-103), la CCC asume el riesgo del cliente, se compromete a pagar al banco del exportador si su cliente exterior se declara en quiebra. La Farm Bill de 1996 fijó el presupuesto global de garantía de crédito en $\$ 5500$ millones USD al año; Otros mil millones suplementarios serían reservados para la asistencia técnica y financiera en los mercados emergentes - Emerging Markets Program-.

El aumento en los precios, especialmente en los cereales, junto con el del monto en los subsidios, permite que los agricultores multipliquen sus ingresos. Los subsidios públicos representaron $115 \%$ de la producción en 2004. La Ley Agrícola o Farm Bill, aprobada el 15 de mayo de 2002 por el presidente Bush, contemplaba un presupuesto total de \$150 mil millones USD, un aumento de casi $80 \%$ con respecto a las disposiciones de la Farm Bill anterior, la Fair Act que rigió desde 1996 hasta 2002 (IATP, 2007b; 2007c; 2007d). 
De esta forma, los Estados Unidos concilian libre comercio, subvenciones a la exportación y proteccionismo: el primero, ideal para productos mediante los cuales han conseguido situarse en posición de líder mundial, las segundas, requeridas en sectores en donde otros las practican, y el tercero, esencial cuando la producción nacional necesita ser defendida. ${ }^{3}$

\section{La ayuda alimentaria.}

La creación de la ayuda alimentaria para países "en desarrollo" en 1954, gracias a la Public Law PL-480 del presidente republicano, el general Dwight Eisenhower, es el primer intento en conquista de los mercados del "tercer mundo" por medio de la oferta de cereales en forma gratuita. Pioneros en la ayuda alimentaria, los EEUU la utilizan como una herramienta económica internacional, social, cultural y política (IATP, 2007, p. 6).

En el corto plazo, la ayuda alimentaria puede lograr un ahorro significativo en el almacenamiento de productos agrícolas a través de la colocación de excedentes en el extranjero. De esta forma los Estados Unidos organizan la ayuda internacional por medio de la entrega de alimentos, pues es menos oneroso exportar productos agrícolas en el marco de dicha ayuda que pagar por su almacenamiento. Este criterio aparece, hasta la década de 1970, como la piedra angular de la política de ayudas. Dispositivo que además constituye una importante fuente de beneficios para muchas instituciones privadas de transporte de productos alimenticios (Murphy, 2005).

En el largo plazo, el programa Food for Peace de la Public Law 480 abre oportunidades a la exportación y, con los años, los antiguos beneficiarios de la ayuda se han convertido en los principales socios comerciales (Corea del Sur, Israel). La ayuda facilita un cierto desarrollo económico al mismo tiempo que impone nuevos hábitos de consumo. Los alimentos tradicionales: arroz, productos a base de maíz local o yuca, son sustituidos gradualmente por la harina de trigo que representa el grueso de la ayuda alimentaria. La agricultura de los países "en desarrollo" adopta así los modelos del país donante.

Durante la Guerra Fría, quienes comerciasen con Cuba o Vietnam del Norte no tenían derecho a la ayuda citada ${ }^{4}$. En la era de la globalización, los países de la periferia figuran como un mercado fabuloso por conquistar y la ayuda alimentaria es el pretexto para penetrar todas las áreas del mundo (Le Monde, 2008). Desde hace veinte años, las donaciones se concentran en África con el objetivo de garantizar el suministro de materias primas por medio de la creación de lazos políticos y comerciales (IATP, 2007e).

En Bolivia, se evaluó el Programa de donación financiado por USAID y los resultados mostraron que una mezcla de maíz y soja tenía en-
3. La Ley Arancelaria de 1930 es una legislación contra la competencia desleal -dumping incluido-. Previa consulta a la Comisión de Comercio Internacional y a la

Administración de Comercio Internacional, el gobierno de los EE.UU. puede tomar medidas de represalia contra los países culpables de tales actos. Sin embargo, se prevén formalmente excepciones para las importaciones procedentes de países económicamente periféricos.
4. En 1996 los EE.UU. aplican una estrategia similar: con las leyes "Helms-Burton" y "D'AmatoKennedy" el Congreso manifiesta la intención de imponer "sanciones" no sólo contra Cuba, Irán y Libia, sino también en contra de nacionales de estos países y en contra de sus socios, incluso en ausencia de cualquier vínculo con los EE.UU. 
tre 3 y 10\% de soja genéticamente modificada. Pero el caso de Colombia fue más crítico, al evaluarse el Programa de ayuda alimentaria que realiza el Instituto de Colombiano de Bienestar Familiar (ICBF), entidad que distribuye en las escuelas y hogares infantiles de la población más pobre del país, alimentos con un alto contenido de soja. Esta acción, realizada por el Grupo Semillas y Consumidores de Colombia COCO, reveló que el $90 \%$ de esta soja era transgénica, correspondiente a la variedad soja Roundup Ready de Monsanto resistente al herbicida glifosato (Vélez, 2003. p. 22 y 23).

Hoy en día, los EEUU controlan casi la mitad de la producción mundial de granos y oleaginosas y optan por la lucha continua para el desarrollo de mercados para la exportación (Market Access for Agriculture) - tal como indica Lilliston (2007) - pues esto es, en todo caso, más eficaz para su economía que las decisiones de embargo o los acuerdos internacionales para estabilizar los precios de los productos básicos. Los farmers estadounidenses saben por experiencia que el arma alimentaria existe y que la mejor manera de utilizarla es seguir produciendo al mejor precio y ser siempre los más rápidos para satisfacer la creciente demanda mundial (IATP, 2007e).

\section{La Unión Europea}

La Unión Europea, primer importador y segundo exportador de productos agroalimenticios, ocupa con los Estados Unidos un lugar central en la producción y comercio mundial. La política comercial europea es altamente determinada por la agricultura, en gran medida porque el mercado común agrícola está integrado al nivel de la competencia comunitaria (Bianchi, 2010).

En el contexto europeo, Francia juega un papel clave, sea cual sea el criterio escogido para medir su importancia, superficie agrícola utilizada (SAU) 3141 800 ha (Hudault, 1993), número de bovinos adultos, rendimientos en cereales y en oleaginosas, exportaciones dentro y fuera de la Unión, o en relación con la participación en el presupuesto agrícola, "la participación es preeminente de Francia en el gasto agrícola de la Unión Europea. De un total de 40,6 mil millones [de euros] recibe 22,5\%, seguida de Alemania, Italia, España y Gran Bretaña (14,2\%; 12,5\%; 11,3\%; 10, 8\%, respectivamente)" (Revel, 2007, p. 8). 


\section{La Política Agrícola Común europea (PAC) de 1962 a 1980}

Para la Comunidad Económica Europea (CEE) al momento de su creación en 1957, así como para la mayoría de países de la Organización para la Cooperación y el Desarrollo Económico (OCDE), el objetivo primordial de las políticas agrícolas nacionales era mejorar la autosuficiencia alimentaria, necesidad ligada a las tensiones políticas provocadas por la Guerra Fría y a la deficitaria balanza comercial agroalimentaria. En este contexto, los fundadores de la CEE en 1957 y de la PAC en 1962 dieron una importancia estratégica a la independencia frente a la importación de alimentos, en particular en el sector de los cereales (Quatremer, 2008).

La autosuficiencia planteada contemplaba una política combinada de oferta de productos y de estímulo a la producción. La PAC de 1962 establecía como instrumento idóneo la regulación de los precios, con lo cual se protegía el mercado interno regulado mediante stocks y exportaciones subvencionadas. El instrumento institucional más importante recibirá el nombre de Fondo Europeo de Orientación y de Garantía Agrícola (FEOGA), con el que se establecerán las "c) Medidas comunes adoptadas para alcanzar los objetivos definidos en el artículo 39 párrafo 1 inciso a) del Tratado " (Bianchi, 2006, p. 12 y 455). ${ }^{5}$

Sobre esta base, la CEE establece para los cereales y las carnes un sistema de precios que se mantienen dentro de un rango específico, asimismo una subvención acordada considerando la diferencia entre el precio mundial y un precio "mínimo" umbral que protege el mercado interior (Hudault, 1999). Este mecanismo de apoyo a los precios y, mediante él, la garantía de los ingresos de los agricultores se complementa con una serie de políticas estructurales y sociales que contribuirán a la postre al mejoramiento del rendimiento de la agricultura europea: incentivos para la modernización, compensación de desventajas naturales, disposición a numerosas innovaciones técnicas (investigación, extensión). El contexto de la década de 1960 fue así particularmente propicio para el rápido desarrollo de la agricultura, hecho que se concretiza en la autosuficiencia y control del mercado interno europeo, estabilizando al mismo tiempo los precios a un nivel razonable para consumidores e industriales (Blumann, 1996; Huiban, 2003).

\section{Una máquina de producción fuera de control}

En la década de 1980 muchos de los indicadores pasan al rojo. Para esta época se es testigo de los efectos de la concentración y la especialización: explosión de los gastos presupuestales, estancamiento, disparidades en los ingresos que privilegian la gran producción (The Economist, 2004) y la aparición de excedentes estructurales acumulados en stocks invendibles (Quatremer, 2008). Todo indicaba que la máquina productiva estaba fuera de control,
5. Para cumplir con la financiación de la Política Agrícola Común, el FEOGA fue establecido por el Reglamento N. ${ }^{\circ} 25$ de 1962 (modificado por el Reglamento CE N. ${ }^{\circ}$ DO $1290 / 2005$ L 209 de 11.8.2005, p. 1), este dispositivo constituye y absorbe actualmente más de $40 \%$ del presupuesto general de la Unión Europea. 
"la Comunidad se ha vuelto autosuficiente en la mayoría de los productos agrícolas y su grado de autosuficiencia alcanza en algunos sectores proporciones alarmantes" (Blumann, 1996, p. 43). Superávit que se acompaña de la dependencia crónica de importaciones masivas necesarias para la alimentación animal, suministros en soja provenientes de Estados Unidos y de Brasil (Blumann, 1996).

\section{PAC 1980-1992: un necesario flujo de excedentes agrícolas hacia una política comercial}

Europa padece en los años ochenta de un superávit estructural, lo cual produce en esa década una serie de ajustes con el fin de intentar controlar la expansión de la producción y los crecientes costos fiscales: retirada de tierras cultivables, cuotas lácteas (Bianchi, 2004), cantidad máxima garantizada (CMG), extensificación, y otras medidas agroambientales de naturaleza imperfecta (Hudault, 1999, pp. 100-103); sin embargo, estas acciones fueron incapaces de frenar la superproducción acusada al distorsionar los precios en el mercado mundial. Las "colinas de trigo" o las "montañas de mantequilla y leche en polvo" al no encontrar compradores en Europa del Este tomaban forma de ayuda alimentaria. La conquista de los mercados extranjeros se convirtió entonces en una necesidad.

Ante esta situación, el objetivo de la seguridad alimentaria europea es complementado por la búsqueda de mercados externos. Desde entonces, Europa, proteccionista en esencia, adopta su segunda naturaleza, la comercialización. La Comunidad Económica Europea participa de esta forma, desde la década los ochenta, en los mercados de América Central y África del Norte, iniciando una lenta regionalización de sus exportaciones y concentrándose espacialmente en los "nuevos" mercados de Europa del Este y en los más ricos de la OCDE. En efecto, a pesar de sus muchas disputas comerciales, Europa continuó siendo el principal proveedor de los Estados Unidos, así como su principal cliente (Blumann, 1996).

\section{La PAC de 1992: reforma bajo presión internacional}

Entre 1980 y 1990 , el total de las exportaciones agroalimentarias europeas se duplican ocupando el segundo lugar a nivel mundial. El aumento de los excedentes y la creciente competencia entre Europa y los Estados Unidos de América, configuran el contexto propicio para el cuestionamiento externo de los mecanismos de la PAC. De esta manera, el componente comercial de la PAC se hace insostenible frente a las negociaciones multilaterales de 1986 durante la Ronda de Uruguay, en el marco de las rondas de negociaciones para la liberalización del comercio mundial del General Agreement Tariffs and Trade (GATT), que culminan con la creación de la Organización Mundial del 
Comercio (OMC). Desde ese momento se incluyen en el marco de este nuevo acuerdo de libre cambio los productos agrícolas que hasta entonces figuraban como ausentes (Hilf, 2005).

En consecuencia, la intervención del Estado para regular el sector agrícola es reducida teóricamente sobre la base de las tesis liberales según una clasificación tripartita, que corresponde a la afectación del tipo de ayudas sobre el mercado internacional. Fueron así designadas las ayudas estatales en diferentes niveles: naranja, azul y verde, las primeras denominan aquellas totalmente prohibidas formalmente, como la regulación por medio de subvenciones para el control de precios o aquellas otorgadas a los sectores exportadores; pasando por las que deben desaparecer a término, hasta las permitidas(de carácter ecológico o de tipo estructural).

De esta manera, las acciones de los estados: regulación de precios, constitución de stocks y, en consecuencia, la producción de excedentes son, desde esta nueva perspectiva, las principales barreras al flujo y al aumento del comercio internacional, pues ellas generan fallas en el mercado, que según David Ricardo se convierte en la principal fuente del bienestar global. Empero, son múltiples las críticas a la aplicación de la citada teoría al mercado de productos agrícolas, además del carácter inflexible de la demanda y del carácter inestable de la producción agrícola sujeta al clima. Sobre esto, Boussard (1987) advierte que:

No es un ejemplo "agrícola" el que Ricardo ilustra en su famosa explicación acerca de las ventajas comparativas en el comercio; al mostrar el interés mutuo que Portugal e Inglaterra tenían en el intercambio de tela y vino, él tenía en mente un esquema del tipo de los que ahora llamaríamos "norte-sur". Aquel en donde se da el intercambio de productos agrícolas provenientes de la "periferia" a cambio de productos manufacturados en el "centro" [...] Von Thünen a diferencia de Ricardo, [...] elimina para comenzar cualquier efecto de ventaja comparativa, razonamiento basado en un modelo en el que una ciudad particular es el único lugar del consumo de productos agrícolas producidos en una amplia llanura homogénea y plana. En estas condiciones, la búsqueda del menor costo para los consumidores y la competencia entre los productores conllevan a ubicar la producción en círculos concéntricos alrededor de la ciudad [...] nos podemos preguntar si esta explicación de la ubicación de los cultivos no 
es mucho mejor que la de Ricardo [...] Lo original [en Von Thümen] es tomar la tierra en su aspecto "continuo" en lugar de identificar solamente un pequeño número de puntos aislados para la oferta y la demanda de productos agrícolas. (pp. 204-205, 212-214)

\section{Los efectos de una política de exportación}

El crecimiento de la producción europea, las exportaciones, la sustitución de productos y el desarrollo de las producciones en déficit muestra efectos negativos sobre la agricultura de los países "en desarrollo". En particular, las exportaciones subvencionadas a bajo precio que compiten y socavan la producción local en el sur.

Las subvenciones a la exportación constituyen un intolerable dumping y la Unión Europea es el mayor culpable [...] Esta práctica es tanto así criminal que se sabe que los agricultores del sur no son capaces de defenderse de las exportaciones subvencionadas [...] (Berthelot, 2001, pp. 111 y 112).

Estas políticas contradicen los proyectos de ayuda al desarrollo de la producción de alimentos que sostiene Europa en los países periféricos (Lambert \& Cogneau, 2006).

\section{PAC 1999: agricultura multifuncional, diversificación y desarrollo}

La reforma llevada a cabo en 1999 contempla el concepto de multifuncionalidad de la agricultura y la administración de proyectos comunitarios rurales (Détang \& Piguet, 2003). A nivel mundial el concepto se desarrolla lentamente (Torrens, 2004; Murphy \& Santarius, 2007; O’Neill, 2002). Simultáneamente, la relación entre agricultores y otros actores rurales se vuelve indispensable para organizar el territorio y la coexistencia de una agricultura europea cada vez más diversa (Huiban, 2003). También el desarrollo sostenible exige nuevos métodos y una relación diferente con el ambiente, con el contexto social y con el conocimiento.

En el campo ambiental se destacan las exigencias de preservación de los recursos naturales y de la biodiversidad, aunadas a demandas crecientes en términos de riqueza paisajística -"Landschaftsästhetik"- (Hudault, 1995, pp. 65-66), sin olvidar la seguridad y la calidad de los productos. La agricultura 
es así considerada parte de una cadena alimenticia en donde todos los factores son interdependientes (Ballarín, 2007).

Europa asiste desde hace algunos años a una proliferación de modelos de producción que integran el desarrollo de la pluriactividad agrícola. Austria, Noruega y Japón, desarrollan unidades productivas de pequeño tamaño, una agricultura pluriactiva y de tiempo parcial en términos laborales, la cual se acompaña de actividades no agrícolas. La pluriactividad agrícola alcanza $22 \%$ en los Países Bajos, 44\% Alemania, 24\% Francia, 80\% Canadá, 60\% Japón y $70 \%$ en Noruega (Geiger, 2003). Estos modelos, que se desarrollan lentamente, siguen siendo marginales. En efecto, la política de apoyo europeo no contempla estas nuevas tendencias y destina la mayor parte de los recursos a las grandes explotaciones (Hudault, 1999).

La búsqueda de una mayor liberalización del comercio agrícola amenaza por ende la agricultura multifuncional europea (Hilf, 2005). La presión del mundo productivo internacional pesa sobre Europa para que se abra a la competencia, obligando a sus agricultores a aumentar su competitividad, lo que entra en contradicción con los esfuerzos hechos en este continente hacia una agricultura más respetuosa de la naturaleza y de las demandas de los consumidores (Varella, 2002).

\section{PAC 2003}

La reforma de la Política Agrícola Común de $2003^{6}$ es anunciada de manera temprana por el comisario europeo de la agricultura Franz Fischler, quien impulsa una amplia reforma y anuncia, para sorpresa del presidente francés Chirac y del Canciller alemán Schröder, la necesidad de desacoplar las ayudas agrícolas en Europa (The Economist, 2004; Revel, 2007).

El "desacoplamiento" de la política agraria supone el establecimiento de ayudas por medio de derechos a un pago único (DPU) (Blumann, 2004). Estos derechos no están relacionados con una producción específica. De este modo, el agricultor puede decidir libremente lo que quiere producir y seguir recibiendo las ayudas según el histórico de su producción, incluso si el agricultor decide no producir. En cualquier caso, es obligatorio para acceder a dichas ayudas mantener la tierra en "buenas condiciones agrícolas y medioambientales" (Bianchi, 2006). De esta manera, el monto de las ayudas que distorsionan el mercado internacional sería controlado y reducido, lo que coincidiría con las tendencias globales. No obstante, importantes áreas de producción permanecen inmunes a estos imperativos y los niveles de apoyo continúan siendo altos, lo que contribuye a la disminución de los precios mundiales, especialmente en el sector de los cultivos extensivos y de la ganadería (Blumann, 2004). "A la luz de los criterios del Acuerdo sobre la Agricultura [GATT/OMC], la reforma plantea dudas sobre la compatibilidad entre el nuevo
6. Reglamento (CE) N. ${ }^{\circ}$ $1782 / 2003$ del 29 de septiembre de 2003 , que establece normas para los regímenes de ayuda directa e instaura determinados regímenes de ayuda a los agricultores; Reglamento N. ${ }^{\circ}$ $1782 / 2003$ (normas comunes), $1783 / 2003$ (desarrollo rural), 1784/2003 (cereales), 1785/2003 (arroz), 1786/2003 (forrajes desecados), $1787 / 2003$ y $1788 / 2003$ (productos lácteos), DOUE, L 270, 21 de octubre de 2003. 
régimen de ayudas desacopladas y la disciplina en las ayudas establecida por el Acuerdo sobre la Agricultura" (Sant'Ana, 2008, p. 208).

\section{PAC 2009: la revisión de la Política Agrícola Común}

Los reglamentos de 2009 confirman la elección hecha desde el año 1999, cuyo objetivo era la consolidación del primer pilar de la PAC, adoptando una transferencia creciente de fondos presupuestales, concebidos éstos como ayudas directas para el desarrollo rural en términos estructurales. Elementos que deben ser entendidos como una reacción ante la coyuntura económica (García, 2008) y frente a la especulación financiera de productos alimenticios (Kaufman, 2010). Sin embargo, el ámbito de aplicación de estos presupuestos intervencionistas de debe consolidar ya que la orientación hacia el mercado internacional se confirma con solidez (García, 2009).

Los vínculos entre la UE y la Organización Mundial del Comercio (OMC), al igual que entre el derecho comunitario y las normas de la OMC, son numerosos y de vieja data, tanto como las organizaciones mismas. Los ejemplos de esta interacción abundan, en primer lugar las sucesivas reformas de la Política Agrícola Común (PAC). Estas reformas, han conducido a la reducción de los subsidios y al desacoplamiento de las ayudas. Con la reforma de la PAC en 2009 , se confirma una política agrícola cada vez más orientada al mercado y más en línea con las disciplinas de la OMC. (Pingel, 2010, p. 183)

\section{PAC 2013: perspectivas sobre la próxima reforma}

Las reformas de la PAC de los últimos veinte años han buscado adecuarse a las disposiciones del Acuerdo sobre la Agricultura de la OMC, respetando con dificultad los intereses de los agricultores de pequeña y mediana escala y adaptándose de manera deficiente a las demandas de los consumidores o de los ciudadanos en lo concerniente a los objetivos medioambientales (García, 2009).

Ahora bien, frente al panorama de la probable reproducción de las crisis alimentarias mundiales vividas desde 2008, se fortalece progresivamente el consenso en el seno de las grandes organizaciones productoras europeas. Por ejemplo en Francia, la Fédération nationale des syndicats d'exploitants agricoles (FNSEA), según la cual la agricultura europea debe ser tan competitiva a nivel internacional como para participar en el control y suministro del 
mercado mundial, particularmente, en el sector de los cereales y de las carnes (Roberts \& Gunning, 2007; Bianchi, 2010; Bricas \& Daviron, 2011).

Francia ha abogado desde el comienzo de la crisis mundial de alimentos por el relajamiento de las restricciones sobre los productores, para que puedan satisfacer la creciente demanda de productos agrícolas tanto en la Unión como fuera de ella (Blumann, 2009, pp. 9 $-10)$.

En este contexto, y desde una perspectiva global, es válido preguntar ¿cuáles orientaciones puede adoptar el componente comercial internacional de la PAC para establecer un sistema comercial multilateral más equitativo?, ya que el continuo y costoso apoyo público a los grandes agricultores exportadores garantiza que estas economías europeas conserven una ostensible ventaja frente al mundo. ¿En qué condiciones competirían los países en "vía de desarrollo"?, los cuales bajo la presión social propia de economías periféricas y dependientes, y bajo la influencia de instituciones internacionales, liberalizan el acceso a sus mercados en detrimento de la capacidad productiva nacional.

La competitividad que brinda la PAC a los productores europeos, así como las ayudas de la Farm Bill a los productores estadounidenses, incita a producir más y a exportar; esta promoción a la exportación se consolida con la venta de productos agrícolas a precios imbatibles en el mercado internacional.

Los excedentes agrícolas de los Estados Unidos de América y de la Unión Europea han hundido los precios de los mercados mundiales de cereales y de carne, deprimidos de antemano por la donación de alimentos. Los excedentes han ejercido una presión a la baja sobre los precios nacionales en todo el mundo, hecho que ha obstaculizado el desarrollo de la producción en los países llamados pobres o, mejor dicho, empobrecidos por la extracción de materias primas y por el precario desarrollo productivo, países cuyos gobiernos se ven obligados políticamente a preferir los productos importados, sean ellos más baratos que los productos locales, o totalmente gratuitos en forma de donaciones.

\section{América latina}

Los agricultores de América Latina fueron durante décadas un actor social, político y económico importante, vanguardia y esperanza para el cambio social. Los movimientos sociales campesinos se constituyeron en el centro de preocupaciones políticas y de investigaciones académicas (González, 1998). 
Desde entonces, la importancia política de estos movimientos sociales y de las guerrillas campesinas ha disminuido o cambiado de naturaleza; el éxodo rural hacia los suburbios de las grandes ciudades no deja de aumentar de manera desproporcionada. Ya no se evocan las zonas rurales en los medios de comunicación excepto cuando se trata de cultivos ilícitos o riesgos ambientales, pues con frecuencia, las políticas nacionales han subestimado el potencial de estas zonas y en particular el del campesinado.

La reforma agraria en sus diferentes versiones (Adam, 2006), fue en América Latina un grito de guerra para liberales y revolucionarios a lo largo del siglo $X X$ que, desde las tropas de Zapata hasta las guerrillas nicaragüenses, salvadoreñas o colombianas, blandían como objetivo algo que se asemejaba al "campesino medio" norteamericano o europeo. Salvo algunas contadas excepciones en México y en Bolivia, América Latina siempre ha sufrido un proceso inverso que exacerba la polarización entre la gran propiedad terrateniente y la mano de obra agrícola (Feder, 1971).

El retraso de México en comparación con las antiguas colonias británicas de América del norte probablemente se explica por las siguientes razones: [...] d) en la Nueva España, las mejores tierras fueron apropiadas por los hacendados. En Norteamérica, la población blanca podía acceder más fácilmente a la tierra y, en Nueva Inglaterra, las explotaciones familiares eran la norma. Las dificultades de acceso a la tierra en las colonias españolas fueron reconocidas por Adam Smith y por el virrey de Nueva España como un impedimento para el crecimiento económico. Rosenzweig (1963) cita a este último [Revillagigedo] de la siguiente manera: "La mala distribución de la tierra es un gran obstáculo para el desarrollo de la agricultura y del comercio, sobre todo debido a los problemas relacionados con el absentismo o el abandono de parte de los propietarios. Tenemos aquí a sujetos de su majestad, que poseen cientos de hectáreas - lo suficiente para formar un pequeño reino -, pero que no producen mucho de lo que valga la pena" (Maddison, 2001, p. 1).

Hoy en día, los pequeños agricultores son sistemáticamente privados de su derecho a la tierra a medida que la propiedad se agrupa en el seno de vastas explotaciones controladas por propietarios terratenientes locales o multinacionales (Arias, 2012). Este proceso es el producto de una variedad de agen- 
tes dispersos, gobiernos nacionales o extranjeros, fondos soberanos, agronegocios multinacionales, Banco Mundial, FMI (Adam, 2006; Boussard \& Delorme, 2007). Agentes éstos que combinan modernización y neo-liberalismo, cuyo objetivo común es la integración económica mundial (Mesclier, 2001).

En este contexto, se considera que la pequeña agricultura de subsistencia es económicamente atrasada e ineficiente, y no sólo por su precaria capacidad tecnológica y mecánica, sino fundamentalmente debido a la estructura de sus relaciones de intercambio (Forero \& Torres, 2002). El monocultivo orientado a la exportación inevitablemente conduce a la producción a gran escala y a la concentración de la propiedad. La producción de agrocombustibles es un ejemplo capital de este proceso, dirigida tendencialmente a crear un monopolio de la tierra ejercido por inmensas unidades de producción, las cuales emplean regimientos de trabajadores agrícolas que laboran para el mercado global (González, 2012b).

En Brasil, 1\% de terratenientes (fazendeiros) poseen más de $43 \%$ de las tierras, $53 \%$ de campesinos poseen menos de $3 \%$ de las tierras cultivables; y 12 millones de campesinos no tienen tierra, [ésta] es hoy en día entregada al furor del comercio agrícola "moderno"una alianza entre grandes agricultores y una media docena de multinacionales que dominan la agricultura Brasileña (Arbex, 2005, p. 34).

Los damnificados por este proceso se suman a las categorías de pobres que no poseen la tierra suficiente para sobrevivir y que pueblan la periferia de las ciudades sin estado en América Latina (González, 2011). Aquellos que desafían las tendencias y se quedan habitando el espacio rural se someten a relaciones laborales propias de la época colonial, "Un estudio realizado por la Universidad de Araquara (Brasil) muestra que hoy en día el promedio de vida de un trabajador en una plantación de caña de azúcar es más bajo que el de los esclavos en la época colonial" (Papacek, 2008, p. 41). Esta sobreexplotación es concurrente con un proceso de mecanización que sustituye a gran parte de esta población desplazándola a otros sectores productivos. Se constituyen así diversas modalidades de modernización / capitalización multinacional, como el caso de la soja, del maíz, de la palma de aceite o de la caña de azúcar en las diferentes regiones de América Latina, lo que provoca el desalojo de campesinos de grandes extensiones de tierra irrigable y fértil (Arbex, 2005), y a la ampliación de la frontera agrícola con la subsiguiente destrucción de áreas protegidas por disposiciones ambientales o pobladas por comunidades indígenas (Lambert, R. 2006), lo cual genera conflictos frecuentes y violentos (Aublet, 2001). 
A pesar de todo lo anterior, el mundo social rural en América Latina continua existiendo, las relaciones que le dan forma se transforman profundamente, posiblemente más que en los periodos pasados. El aumento ponderado de la población ha sido lo suficientemente sostenido como para contrarrestar la migración hacia las urbes y consolidar un aumento de la población en valor absoluto en las zonas rurales (The Economist, 2005).

La lucha del MST [Movimiento de los Trabajadores sin Tierra] es la historia misma del Brasil. Ningún movimiento de campesinos organizados a escala nacional ha durado tanto tiempo ni ha tejido tantos vínculos profundos con la sociedad civil. EI MST gerencia 1300 escuelas primarias y emplea a 3000 educadores que forman 160000 niños y adolescentes. [...] En enero del año pasado, el MST inauguró la primera Universidad del Pueblo en Brasil, en Guararema, a 60 km de Sao Paulo (Arbex, 2005, p. 34).

\section{Un crecimiento de la producción superior a la media mundial}

Los análisis económicos de América Latina a menudo designan la década posterior a 1980 como la "década perdida", por la instalación de una crisis de la deuda externa en la que se sumieron la mayoría, si no todos los Estados de la región. En el análisis no se ha contemplado la concentración real del sector productivo en la exportación agrícola para las últimas dos décadas del siglo pasado, fenómeno acompañado de conocidas medidas de carácter estructural que convierten a la participación en el mercado internacional y de la no intervención del Estado en los nuevos derroteros.

Tal como fue aplicada a los países en desarrollo, la reforma de la agenda política de orden liberal es conocida como el "Consenso de Washington», un conjunto de reformas políticas, sobre todo de carácter macroeconómico, para hacer que los mercados funcionen mejor (corrección de precios). Dichas reformas incluían el fortalecimiento de derechos de propiedad, la liberalización de los mercados nacionales, la privatización de las empresas estatales y el establecimiento de la apertura de la economía al libre comercio y a la inversión (Hunter, 2006).

En el nuevo siglo, la participación de la región en el mercado internacional evoluciona y pasa de 9,6\% en 2000 a 11,7\% en 2008 (WTO, 2009). Las exportaciones hacia Asia (China), pasan de $14,8 \%$ del total a $20 \%$ en el mismo periodo. 
Brasil se ha convertido en el primer exportador mundial de soja hacia la China, y sus ventas de acero destinadas al Empire du Milieu se han disparado en $500 \%$ en 2003. El etanol, del cual Brasil se hace un especialista, es considerado en China como una alternativa energética de primer orden. Los patrones de la agroindustria "se frotan las manos" porque el presidente Hu Jintao prometió poner fin a las barreras sanitarias que impedían el acceso de pollo y carne del Brasil. Los argentinos también se beneficiarán, además de la soja, podrían vender más carne, cuero, lana, entre otros (Le Figaro, 2004).

En este proceso de crecimiento, Argentina y Brasil son dos modelos notables. El índice de crecimiento de la participación en el mercado internacional de productos agrícolas pasa de 17261 millones de dólares en 1990 es decir 4,16\% del mercado, a 98902 millones de dólares en 2008, representando $7,7 \%$ (WTO, 2009). Este crecimiento, en sectores tales como el de los cereales y plantas oleaginosas, continúa hoy en día para otras grandes plantaciones en toda América Latina pero a un ritmo mucho menos sostenido.

El desarrollo antes mencionado es jalonado, en infraestructura, en producción y en comercialización por poderosos complejos agroindustriales. Agribusiness nacional e internacional que representa $30 \%$ del PIB y un impacto muy positivo en la balanza comercial del Brasil y de la Argentina. En América latina, el MERCOSUR (Argentina, Brasil, Paraguay, Uruguay) aparece como la región agroexportadora por excelencia, pues representa en 2008 \$107 043 mil millones USD del total regional de \$170,9 mil millones. En efecto, Argentina exporta $\$ 37,5$ mil millones de los cuales $\$ 12,8$ mil millones van hacia los Países Bajos, España, Italia y Alemania principalmente. Brasil a su turno exporta $\$ 61,4$ mil millones, de los cuales $\$ 21,7$ mil millones van hacia los Países Bajos, Alemania, Reino Unido y Bélgica, sin incluir la China y el NAFTA (CEI, 2009; OMC, 2009; Toepfer, 2009).

En el caso de Brasil las transformaciones han sido considerables en los últimos cuarenta años; su economía pasa de ser un modelo agro-exportador de materias primas brutas "commodities", a una organización productiva más desarrollada, reflejo del carácter emergente y "semi-industrializado" de su estructura económica. El mundo entero reconoce el peso de Brasil, su capacidad para definir políticas internacionales conforme con sus intereses, además es considerado como un temible competidor en el comercio de productos agroalimentarios. 
7. El maíz está en el corazón de las tradiciones mexicanas desde hace 6000 años, esto explica el apego de los campesinos, a pesar de la competencia de maíz importado a menores precios. En efecto, la autosuficiencia y el control de muchas variedades son necesarios para lograr la preparación de platos tradicionales imposibles de preparar con las variedades importadas.
Aunque el apoyo al sector agrícola disminuye desde los años 90, el sistema brasileño de producción mantiene y perfecciona herramientas de regulación (política de almacenamiento) y de intervención pública (Adam, 2006): crédito, desarrollo de infraestructura, investigación, educación, generación endógena de tecnología; se trata de la intervención del Estado en la creación de condiciones favorables a la producción. Elementos tales son indispensables para corregir las imperfecciones del mercado y generar la competitividad del aparato productivo tanto a nivel interno como a nivel internacional (OCDE, 2007).

[...] Pero este proceso de mecanización de la agricultura brasileña ha dado lugar a la expulsión masiva de campesinos y ha sido uno de los factores decisivos en la conformación del MST junto con la construcción de la represa de Itaipú, construida a partir de 1975 en el río Paraná, frontera entre Brasil y Paraguay. En su construcción, miles de familias se vieron obligadas a abandonar sus tierras y hogares. Estos dos acontecimientos irrumpen en la historia de Brasil como una etapa de modernización tecnológica; pero para miles de pequeños agricultores significó el desplazamiento de sus fuentes de empleo, la expulsión y el desarraigo. Fueron estos expulsados quienes darían nacimiento al MST. (Pellegrini, 2011, pp. 62-63)

El alto rendimiento en la producción de cereales oculta algunas disparidades regionales. Si bien es cierto que la producción total ha aumentado significativamente en Argentina y Brasil, en general, América latina sufre de un déficit creciente en estos productos, lo cual provoca el aumento de las importaciones. Por ejemplo, Colombia de importar $\$ 533$ millones de productos agrícolas en 1990, pasa a importar 4542 millones en 2008 (OMC, 2009). En 2009, también México se convirtió en el mayor importador de maíz a nivel mundial con 7,4 millones de toneladas provenientes en su mayoría de los EE.UU. ${ }^{7}$ (The Economist, 2004; Toepfer, 2009; Charvet, 2010).

El maíz mantuvo sin pausa el brillo de las civilizaciones o semi-civilizaciones inca, maya y azteca, auténticas creaciones de esta planta; para luego realizar un singular éxito a escala mundial. [...] el maíz de regadío cultivado en las terrazas andinas o en las orillas lacustres de la meseta mexicana conduce [...] a estados teocráticos, tiránicos sin medida, y todo este ocio campestre será utilizado para inmensos trabajos al estilo egipcio. Sin el maíz, no hubiesen sido posibles las gi- 
gantescas pirámides de los mayas o de los aztecas, o los muros ciclópeos de Cuzco o las impresionantes maravillas de Machu Picchu. (Braudel, 1979, pp. 172 y 175).

En este contexto regional, la deficitaria producción para el consumo urbano de cereales en países con superficies reducidas, no aptas para su cultivo y sin apoyo para la pequeña producción, está llamado a ser suplido por el mercado internacional. Pero este déficit puede también ser el reflejo de una política de apoyo selectivo a productos de alto rendimiento destinados a la exportación, como la soja en Brasil. En este país el déficit en trigo obligaría a la importación de seis millones de toneladas en 2009 (Toepfer 2009).

La particular liberalización de los mercados latinoamericanos, acompañada de Programas de Ajuste Estructural con el respaldo del Banco Mundial y del Fondo Monetario Internacional, a la cual contribuyen gobiernos y elites nacionales desregulando y abriendo mercados agrícolas (IATP, 2007), constituye lo que la UNCTAD United Nations Conference on Trade and Development denomina una liberalización sin efectos positivos (Adam, 2006, p. 384). Los mercados locales son presa fácil de multinacionales europeas y estadounidenses que venden a pérdida sus productos y así logran deshacerse de la competencia local, incrementando la pobreza y estimulando la concentración de la tierra y la dependencia alimentaria. ${ }^{8}$

El impacto del dumping de los EE.UU. ha contribuido a un descenso en el número de puestos de trabajo disponibles relacionados con la agricultura en México. El empleo agrícola en México es de alrededor de 8,1 millones en la década de 1990 antes del NAFTA y de la Farm Bill de 1996. En 2006, México cuenta con sólo 6 millones de trabajadores, una pérdida de más de dos millones de puestos de trabajo relacionados con la agricultura. La pérdida de empleos agrícolas coincide con una importante migración del campo a las ciudades tanto mejicanas como estadounidenses. El NAFTA y la Farm Bill de 1996 aceleran la tendencia. De 1980 a 1994, la migración desde las zonas rurales mexicanas a los EEUU aumenta en 95\%. Para el año 2002, la migración a los EEUU desde el México rural es de $452 \%$ más alta que en 1980 (Spieldoch \& Lilliston, 2008).
8. El aumento del consumo de las poblaciones latinoamericanas, sobre todo a nivel urbano, favorece la aparición y desarrollo de la presencia de las multinacionales de la distribución. Estos actores internacionales satisfacen la demanda a nivel local en forma de grandes cadenas de distribución que en 1990 representaban $20 \%$ del sector y que en el año 2000 representan $60 \%$. 
9. La complejidad enunciada por este grupo de neologismos, aparece resuelta en Alemania con el término de

"Verrechtlichung". Abogados del campo laboral, desde la izquierda, criticaban el uso del derecho para domestica los conflictos de clase en la República de Weimar. Hoy esta terminología se relaciona con la creciente importancia dada a la forma jurídica para la regulación del nuevo orden global.

10. Habermas analizaba ya en los años ochenta el refuerzo de la racionalidad jurídica pura como el efecto conjunto de la multiplicación de los técnicos del derecho y de su instrumentalización al servicio de finalidades económicas y políticas.
La circulación de productos alimenticios en una economía global engendra una tendencia irresistible de expansión, de apropiación, de homogeneización. Entre más se desarrollan las necesidades de consumo y de distribución, más se consolidan la riqueza y la concentración, proceso acompañado por acuerdos comerciales tales como el de la OMC. Ver también este proceso con mayor profundidad en tesis doctoral del autor de este artículo titulada Le produit agricole et agroalimentaire dans la mondialisation des échanges: contribution à la recherche d'une justice économique internationale (González, 2012a).

En efecto, una de las ideas que articula nuestra reflexión, y de la cual sólo formularemos aquí algunas pistas previas, es la hipótesis de la relación constitutiva entre el hecho económico y el hecho jurídico, entre los procesos de transformación económica internacional y su regulación por medio del nuevo orden jurídico global materializado en parte en los acuerdos de la OMC.

Esta relación constituye aún hoy, para economistas y juristas, un interrogante embarazoso que se resolvería en un análisis que contemplase algunos temas transversales: la tendencia hacia la juridización (Commaille, 2000) del orden internacional, en particular la «tendencia jurisdiccional» de la OMC (Ruiz Fabri, 2003, p. 904), tendencia que testimonia un desarrollo paralelo de juridización a nivel global (Joerges \& Petersmann, 2006) ${ }^{9}$. De tal suerte, la arena jurídica y jurisdiccional global remplazaría con frecuencia a la arena político económica (Habermas, 1987) ${ }^{10}$. Este fenómeno marca a su vez la interdependencia entre la economía y el derecho y nutre simultáneamente ambos campos de estudio (Teubner, 2011).

Ante esto, es necesario también reflexionar sobre el paso de un mundo regulado por un derecho económico internacional de carácter interestatal, hacia la constitución, aún incoherente, de un sistema jurídico mundial, reflejo de transformaciones en las relaciones económicas transnacionales y reflejo del problemático cruce de diversas lógicas para conformar el derecho internacional; entre otras, la orientación anglo-norteamericana, pragmática y flexible, que campea como "reina y señora" (Hudault, 2008).

La soberanía y el territorio son aún características claves del sistema internacional. Pero ellas fueron reconstituidas y, en gran parte, desplazadas hacia otros escenarios institucionales fuera del Estado y fuera de la estructura territorial nacional [...] la soberanía fue descentralizada y el territorio parcialmente desnacionalizado (Sassen, 1996, pp. 29-30; ver también Moncayo, 2004).

Ahora bien, si la tendencia continúa, el futuro y la supervivencia de los hogares rurales en Latinoamérica están fuera de las consideraciones de esta nue- 
va forma jurídica global. Lejos del consenso por parte de las instituciones internacionales sobre la obligatoriedad de los derechos sociales contemplados en las diferentes declaraciones de derechos humanos en relación con la seguridad alimentaria, los sectores sociales concernidos difícilmente pueden anticipar las consecuencias de los procesos de liberalización del comercio de productos agrícolas.

Por esto, desde la perspectiva acogida, la importancia otorgada al rendimiento del mercado no puede omitir los valores colectivos, la relación entre estas dos racionalidades es una cuestión fundamental que no se puede dejar a merced de la lógica mercantil (Jacquot, 2010). Es necesario por tanto considerar la forma de dar alcance material a los valores a partir de los cuales damos tratamiento a la paradojas político económicas de nuestra época (Teubner, 2003).

Tratándose del sector agroalimentario, los objetivos establecidos son cruciales en la posición de la ciudadanía y cristalizan percepciones que inspiran el diseño de una jerarquía en la defensa de la agricultura y de su función principal: la alimentación en términos de cantidad, de disponibilidad y de calidad (Frison, 2006). Esta aproximación jerarquizada a los objetivos debe examinar también los efectos de las actividades agrícolas extensivas sobre el medio natural y social (Hudault, 2008), pues es a partir de una percepción holística que se establece la distinción normativa entre precio y dignidad (Boussard \& Delorme, 2007).

La agricultura campesina en las regiones relegadas del sistema mundial sufre hoy en día de los embates de los procesos de desregulación en boga, en la medida que ella difícilmente resiste a las transformaciones de la agricultura moderna. Los pequeños agricultores constituyen así uno los sectores más desfavorecidos del planeta y estarían condenados a desaparecer de sus territorios, diluidos en la multitud de pobres del sistema mundial. Es necesario por tanto "hacer reemerger" la agricultura campesina en un esfuerzo de recomposición, mediante un enfoque regional enmarcado por formas organizativas que reflejen el carácter común y colectivo del trabajo humano. Se trata entonces de fomentar acciones locales y en red para lograr transformaciones globales.

Asimismo, refutamos la noción de que la globalizaron haya logrado la homogeneización cultural planetaria, porque ello negaría la posibilidad y el derecho de los pueblos para resimbolizar y resignificar su entorno y los diferentes imaginarios, mercancías e ideas que la globalización impulsa. Cada grupo cultural posee un referente simbólico general y escalas de valores y posiciones que se han heredado, re- 
producido y construido, y que se manifiestan a través de prácticas en su cotidianidad: éstos se enfrentan y coexisten con los procesos de globalización, por medio de encuentros, desencuentros, conflictos, disidencias, simbiosis, coincidencias, reivindicaciones; es decir, resignificaciones que otorgan nuevos contenidos a ese referente cultural primero. Además, nos parece importante mencionar la preocupación de que se visualice positivamente la opción integradora mundial como el asumir los patrones e ideas del proceso de globalización, negando, desechando las otras opciones. Esto es lo peligroso de un proyecto globalizador cuyos fines no son la humanización, la solidaridad y el respeto entre unos y otras, sino los intereses político económicos, que buscan la acumulación del capital a costa de otros (Mora \& Solano, 2004, p. 19).

\section{Bibliografía}

Adam, E. (2006). La politique agricole face aux enjeux internationaux: la libéralisation conventionnelle du commerce international des produits agricoles. Tesis no publicada de derecho, Panthéon-Sorbonne, Paris I.

Arias, W, (2012, junio 17). Entrevista Radio Universidad Nacional de Colombia. Programa Debates económicos.

Arbex, J. (2005, julio). L’autre Brésil. Courrier International. 766, 34.

Association Française de Droit Rural AFDR. (2011, 3er trimestre). La lettre du droit rural, Bulletin de liaison de l'AFDR. 40.

Aublet, A. (2001, octubre/diciembre). Mouvements sociaux au Brésil. Regards sur les Amériques. 6, 21-22.

Ballarín, A. (2007, septiembre 26-29). Commission I, Rapport individuel. En XXIVe Congrès et Colloque Européens de Droit Rural. Caserta (Napoles).

Berthelot, J. (2001). L'agriculture talon d'Achille de la mondialisation. L'Harmattan. 
Bianchi, D. (2010). Le rôle de la comitologie (1958-2010) dans la Politique agricole commune (PAC). Ou la chaîne de transmission du droit communautaire agricole. Tesis no publicada de derecho, PanthéonAssas, Paris II.

Bianchi, D. (2008). Politique Agricole Commune. JurisClasseur Europe. Fasc. $1312,40-41$.

Bianchi, D. (2006). La politique agricole commune (PAC). Toute la PAC, rien d'autre que la PAC!. Bruylant.

Bianchi, D. (2004, diciembre). Trente ans d'un régime temporaire. Les quotas laitiers dans la PAC: 1984-2014. Revue de Marché Commun de l'Union Européenne, RMCUE. 483, 655-674.

Blandford, D., Laborde D. \& Martin W. (2008). Implications of the February 2008 WTO Draft Agricultural Modalities for the United States. ICTSD.

Blumann, C. (2009). Quelles politiques communautaires ? la PAC et le budget européen a l'horizon 2013. Annuaire français de relations internationales. $X$. Centre Thucydide - Analyse et recherche en relations internationales.

Blumann, C. (2004). La réforme de la Politique agricole commune. Cahiers de Droit Européen. 40(3-4), 297-335.

Blumann, C. (1996). La politique agricole commune, droit communautaire agricole et agroalimentaire. Litec.

Bonnamour, J. (2006 15 octobre). L'Avenir du Système Agricole et Alimentaire Mondial. Documento de trabajo interno de l'Académie d'Agriculture de France preparado para la sesión del 22 de noviembre de 2006.

Boussard, J. \& Delorme, H. (2007). La régulation des marchés agricoles internationaux. Un enjeu décisif pour le développement. L'Harmattan.

Boussard J. (1987). Économie de l’agriculture. Économica.

Braudel, F. (1979). Civilisation matérielle, économie et capitalisme, XVeXVIIle siècle, 1. Les structures du quotidien. Paris: Armand Colin.

Bricas N. \& Daviron B. (2011, diciembre). De la hausse des prix au retour du productionnisme. Les enjeux du sommet sur la sécurité alimentaire de juin 2008 à Rome. Courrier de l'environnement de l'INRA. 61, 21-26. 
Centro de Economía Internacional CEI. Estadísticas. Recuperadas mayo 26, 2010 de http://www.cei.gov.ar/html/mercosur.htm

Charvet, J. (2010, septiembre). Atlas de l'Agriculture. Comment pourra-t-on nourrir le monde en 2050 ?. Paris: Autrement.

Commaille, J. (2000). La juridicisation du politique. LGDJ y Réseau Européen Droit et Société.

Détang, C. \& Piguet, V. (2003, diciembre). Les ruraux en 1999 : qui sont-ils et d'où viennent-ils?. Recherches en Économie et Sociologie Rurales, INRA. 1-2(03).

Pisani, D. (1984). From the Family Farm to Agribusiness: The Irrigation Crusade in California and the West, 1850-1931. University of California Press, Berkeley.

Engels, F. \& Marx, K. (2002, abril 9) La guerre civile aux États-Unis (18611865). Version digital preparada por J. Tremblay, Québec: Chicoutimi. Recuperada marzo 13, 2009 de http://pages.infinit.net/sociojmt

Feder, E. (1971). The Rape of the Peasantry: Latin America's Landholding System. New York: Anchor Books.

Forero, J. \& Torres, L. (2002). La part des producteurs familiaux dans le système alimentaire colombien. Cahiers des Amériques Latines. 40(2).

Frison, M. (2006, mai 12). Pourquoi une régulation juridique du secteur agricole est-elle justifiée ?. Momagri, Mouvement pour une organisation mondiale de l'agriculture. Recuperado abril 13, 2010 de http://www.momagri.org/FR/articles/Pourquoi-une-regulation-juridique-dusecteur-agricole-est-elle-justifiee-_34.html

García, T. (2009, mayo). La PAC après son "bilan de santé". Revue du Marché commun et de l'Union européenne. 528, 301-311.

García, T. (2008, febrero). Le bilan de santé de la Politique agricole commune. Revue du Marché commun et de l'Union européenne. 515, 7379.

González, L. (1998). Para capturar el derecho. Historia del derecho. Comentarios acerca de la forma jurídica, propiedad de la tierra $y$ movilización social en Colombia. Herramientas para un análisis genealógico. Tesis no publicada. Universidad Nacional de Colombia, Facultad de derecho, ciencias políticas y sociales. Santafé de Bogotá. 
González, L. (2012a). Le produit agricole et agroalimentaire dans la mondialisation des échanges : contribution à la recherche d'une justice économique internationale. Universidad Panthéon Sorbonne, 18 janvier 2012, director de investigación Pr. Dr. M. Joseph Hudault.

González, L. (2012b). Agropaliwa - zarys problematyki prawnej. Przeglad Prawa Rolnego. Agricultural Law Deaprtement and Law Faculty of Adam Mickiewicz University in Poznan Poland. 1(10). Articulo por aparecer en 2012.

González, M. (2011). Les moyens audiovisuels et les communautés indigènes : Diverses pratiques participatives à Bogotá. Femmes et insertion sociopolitique. Tesis no publicada, EHSSS Paris.

Geiger. (2003). Droit de l'agriculture et des filières agroalimentaires. En Notas de curso DESS 2002-2003. Panthéon-Sorbonne, Paris I.

Habermas, J. (1987). Theorie des kommunikativen Handelns, Band 2, Zur Kritik der funktionalistischen Vernunft. Frankfurt am Main: Suhrkamp, IV ed.

Hilf, M. (2005). WTO-Recht. Nomos, Baden-Baden.

Hudault, J. (2008, décembre 10). Du droit agraire au dorit agro-industriel. Académie d'Agriculture de France. Publicada en Revue de droit rural. (2008, noviembre). 367, 11-14.

Hudault, J. (1999). Droit de produire et environnement à travers l'évolution de la PAC. En 40 anni di Diritto agrario comunitario, Atti del convegno, Martina Franca, 12-13 giugno 1998, Guiffrè editore.

Hudault, J. (1995). La multifonctionnalité de l'agriculture, Rapport de synthèse, CEDR, 13-16 octobre 1993. Rivista di diritto agrario. Anno LXXIV, 63-74, Guiffrè editore. Artículo editado posteriormente en L'agriculture multifonctionnelle : aspects juridiques : XVIle congrès européen de droit rural CEDR, 13-16 octobre 1993, Interlaken (Suisse) : (mise à jour novembre 1998) / Comité Européen de Droit Rural, (1999). Paris : L'Harmattan, 709-719.

Hudault, J. (1993, abril 28). L'entreprise agricole face à l'évolution de la législation en France et dans la CEE. Comptes Rendus de l'Académie d'Agriculture de France. 79(4), 3-18.

Huiban, J. (2003, diciembre). Entreprises et activités industrielles en milieu non urbain bilan et perspectives. Espaces ruraux et aménagement du territoire. Recherches en Économie et Sociologie Rurales, INRA. 1-2(03). 
Hunter, R. (2006). Wade the World's Social Inequalities, Should we worry about income inequality ?. International Journal of Health Services, 36(2), 271-294.

Institute for Agriculture and Trade Policy IATP. (2007a). A Fair Farm Bill for the World.

Institute for Agriculture and Trade Policy IATP. (2007b, febrero 23). The Farm Bill and Public Health: An Overview.

Institute for Agriculture and Trade Policy IATP. (2007c, mayo). Une Farm Bill plus juste pour le monde.

Institute for Agriculture and Trade Policy IATP. (2007d). Le Farm Bill américain et la Politique Agricole Commune.

Institute for Agriculture and Trade Policy IATP. (2007e). A Farm Bill for the World's Hungry.

Jacquot, M. (2010). L'OMC dispose-t-elle des moyens pour réglementer les marchés agricoles ?. Académie d'Agriculture de France.

Joerges, C. \& Petersmann, E. (2006). Constitutionalism, Multilevel Trade Governance and Social Regulation. Oxford and Portland.

Kaufman, F. (2010 julio). The Food Bubble. How Wall Street starved millions and got away with it. Harpers Magazine, 27-34.

Lambert, S. \& Cogneau, D. (2006). L'aide au développement et les autres flux Nord Sud: complémentarité ou substitution. Centre de développement de l'OCDE, Document de travail, 251.

Lambert, R. (2006, enero). Main basse sur les terres du Paraná brésilien. Le monde diplomatique.

Le Monde. (2008, mayo 16). Les États-Unis annoncent la reprise de leur aide alimentaire à la Corée du Nord.

Le Figaro. (2004, noviembre 13). Quand la Chine «envahit» l'Amérique Latine.

Lilliston, B. (2007, mayo 13). Farm Bill Perpetuates Market Deregulation and Volatility. Small Gains in Bioenergy, Conservation, Local Foods, Food Aid. Institute for Agriculture and Trade Policy. 
Maddison, A. (2001, octubre 23). L'économie mondiale: une perspective millénaire. En Débat présidé par Christian de Boissieu, salle de la présidence $n^{\circ} 1$. Université Panthéon-Sorbonne, Paris I.

Marx, K. (1953). Grundrisse der Kritik de politischen Ökonomie, Rohentwurf 1857-1858. Berlin : Marx-Engels-Lenin-Institut Moskau, Dietz Verlag.

McDonald, D. Nair, R. Podbury, T. Sheldrick, B. Gunasekera D. \& Fisher B. (2006, septiembre). US Agriculture Without Farm Support, ABARE Research Report 06.10, Canberra.

Mesclier, E. (2001, octubre-diciembre). Décentralisation et monde rural. Regards sur les Amériques. 6.

Moncayo, V. (2004). El Leviatán derrotado, reflexiones sobre teoría del Estado y el caso colombiano. Bogotá: Norma.

Mora, D. \& Solano, V. (2004). Cuando son los «otros» los que hacen antropología. En C. Araya Jimenez \& M. Bolaños Arquin (dirs). Retos y perspectivas de la antropología social y la arqueología en Costa Rica a principios del siglo XXI. Editorial de la universidad de Costa Rica.

Murphy, S. \& Santarius, T. (2007, noviembre). The World Bank's WDR 2008: Agriculture for Development. Response from a Slow Trade - Sound Farming Perspective. Ecofair trade dialogue, Discussion Papers. English Version, Heinrich Böll Fundation, 10.

Murphy, S. (2005, noviembre 17). Food Aid: What Role for the WTO. IATP. Recuperado junio 13, 2008 de http://iatp.org/iatp/publications.cfm? account|D=451\&ref|D=77567.

OCDE. (2007). Politiques agricoles des pays non membres de l'OCDE : suivi et évaluation 2007.

Nadal, A. \& Wise T. A. (2004). The Environmental Costs of Agricultural Trade Liberalization: Mexico-U.S. Maize Trade Under NAFTA. Working Group Discussion Paper DP04, Working Group on Development and Environment in the Americas.

Oliveira, V. (2006, Marzo). The Food Assistance Landscape. U.S. Department of Agriculture, Economic Research Service. Recuperado julio 26, 2009 de http://www.ers.usda.gov/Publications/eib6-2/

O'Neill, M. (2002, junio 28). Agriculture, the EC and the WTO; a legal critical analysis of the concepts of sustainability and multifunctionality. Paper accepted for publication by the Environmental Law Review. 
Papacek, T. (2008, enero). Die „Grüne Macht“. Latein Amerika Nachrichten. 403, Berlin.

Pellegrini, P. (2011, marzo 29). Agriculture transgénique: modes de production et utilisation de la connaissance scientifique. Science, État et Industrie dans les cultures transgéniques en Argentine. Tesis no publicada de ciencias sociales y filosofía del conocimiento, Universidad Sorbonne - Paris IV.

Pingel, I. (2010). L'Union européenne et les négociations agricoles à l'Organisation mondiale du commerce. En La PAC en mouvement. Évolution et perspectives de la Politique Agricole Commune. París: L'Harmattan.

Quatremer, J. (2008, marzo 14). Les paysans dans la rue pour leur blé. Liberation.

Revel, A. (2007, septembre 26-29). Nouvelle PAC, Nouvelle OMC, Nouvel Equilibre.

Régional Européen et Mondial. En XXIVe Congrès et Colloque Européens de Droit Rural - CEDR Commission I, Rapport national, France. Caserta (Napoles).

Revel, A. \& Ribaud, C. (1981). Les États-Unis et la stratégie alimentaire mondiale. Calmann-Lévy.

Rist, G. (2007). Le développement: histoire d'une croyance occidentale. Sciences Pô.

Roberts, I. \& Gunning, C. (2007 agosto). The European Union's Common Agricultural Policy: An Overview of Reforms. Abare Research Report $07.13^{*}$. Canberra.

Ruiz Fabri, H. (2003). La juridictionnalisation du règlement des litiges économiques entre États. Revue de l'Arbitrage. 3.

Sant'Ana, L. (2008). Les relations entre l'Union Européenne et le Mercosur en matière agricole. Tesis no publicada Paris II Panthéon Assas.

Sassen S. (1996). Losing control ? Sovereignty in An Age of Globalization. New York : Columbia University Press.

Spieldoch, A. \& Lilliston, B. (2008). A Fair Farm Bill and Immigration. Trade and Global Governance Program, I.A.T.P., Communications Part of a series on the 2007 United States Farm Bill. 
Teubner, G. (2011). A Constitutional Moment ? The Logics of 'Hitting the Bottom'. En P.

Kjaer, G. Teubner \& A. Febbrajo, The Financial Crisis in Constitutional Perspective. The Dark Side of Functional Differentiation. Hart.

Teubner, G. (2003). Der Umgang mit den Rechtsparadoxien: Derrida, Luhmann, Wiethölter. En C. Joerges \& G. Teubner. Rechtsverfassungsrecht, Recht-Fertigung zwischen Privatrechtsdogmatik und Gesellschaftstheorie. Nomos Verlagsgesellschaft, Baden-Baden.

The Economist. (2005, febrero 17). Down on the farm.

The Economist. (2004, mayo 24). Nostalgie de la boue.

Thoyer, S. (1998). L'économie politique des soutiens à l'agriculture dans les pays développés - le renouveau des politiques agricoles américaines et européennes révèle-t-il un nouvel équilibre?. En G. Miclet, L. Sirieix \& S. Thoyer. Agriculture et alimentation en quête de nouvelles légitimités. Économica, 267-351.

Toepfer International. (2009). Market Review October 22. Recuperado diciembre 26, 2010 de http://www.toepfer.com. http://www.acti.de/

Torrens, J. (2004, junio 13 y 14). La multifonctionnabilité de l'agriculture familiale. En Agriculture familiale, agroécologie et développement rural viable au Brésil et en France. Convention association brésilienne d'ONGs (ABONG) et coordination sud. São Paulo.

UNICEF Innocenti Research Centre. (2006). Child Poverty in Rich Countries, 2005, Part I. International Journal of Health Services. 36(2) 235-269.

Vargas, L. (2006, mayo 16). ¿Europa en crisis o crisis del neoliberalismo?. En Revista Pueblos. Recuperado mayo 13, 2012 de http://www.revistapueblos.org/spip.php?article395

Varella, M. (2002). L'inégalité Nord-Sud et la construction juridique $d u$ "développement durable» dans le droit international. Tesis no publicada de derecho, Universidad Panthéon-Sorbonne, Paris I.

Vélez, G. (2003, octubre 23). Los cultivos y los alimentos transgénicos en Colombia. En Revista semillas. Recuperado septiembre 28, 2012 de http://www.semillas.org.co/sitio.shtml?apc=p1e1--\&x=24230

WTO. (2009). International trade statistics, merchandise trade by product. Recuperadas diciembre 26, $2010 \quad$ de 
http://www.wto.org/english/res_e/statis_e/its2009_e/its09_merch_trade_p roduct_e.htm 